\title{
THE PERIGEO PROJECT: INERTIAL AND IMAGING SENSORS PROCESSING, INTEGRATION AND VALIDATION ON UAV PLATFORMS FOR SPACE NAVIGATION
}

\author{
P. Molina ${ }^{\text {a }}$, E. Angelats ${ }^{\mathrm{a}}$, I. Colomina ${ }^{\mathrm{a}}$, A. Latorre ${ }^{\mathrm{b}}$, J. Montaño ${ }^{\mathrm{b}}, \mathrm{M}$. Wis ${ }^{\mathrm{b}}$ \\ ${ }^{a}$ Centre Tecnològic de Telecomunicacions de Catalunya (CTTC), Castelldefels, Barcelona, Spain - \{pere.molina, \\ eduard.angelats, ismael.colomina\}@cttc.cat \\ ${ }^{\mathrm{b}}$ Aerospace and Defense Department, DEIMOS Space S.L.U., Tres Cantos, Madrid, Spain - \{antonio.latorre, \\ javier.montano,mariano.wis\}@deimos-space.com
}

\section{Commission III, ICWG III/I}

KEY WORDS: Space navigation, image processing, unmanned aerial vehicles

\section{ABSTRACT:}

The PERIGEO R\&D project aims at developing, testing and validating algorithms and/or methods for space missions in various field of research. This paper focuses in one of the scenarios considered in PERIGEO: navigation for atmospheric flights. Space missions heavily rely on navigation to reach success, and autonomy of on-board navigation systems and sensors is desired to reach new frontiers of space exploration. From the technology side, optical frame cameras, LiDAR and inertial technologies are selected to cover the requirements of such missions. From the processing side, image processing techniques are developed for vision-based relative and absolute navigation, based on point extraction and matching from camera images, and crater detection and matching in camera and LiDAR images. The current paper addresses the challenges of space navigation, presents the current developments and preliminary results, and describes payload elements to be integrated in an Unmanned Aerial Vehicle (UAV) for in-flight testing of systems and algorithms. Again, UAVs are key enablers of scientific capabilities, in this case, to bridge the gap between laboratory simulation and expensive, real space missions.

\section{INTRODUCTION}

This paper is framed within the PERIGEO project and focuses on a specific goal: space navigation using inertial and imaging sensors for Earth observation and Atmospheric flight. Its organization is the following: firstly, a description of the project, its testing and validation facilities and a history review of space navigation is provided. Secondly, specific developments in the simulated environment are presented, including preliminary results, and the integration of sensors and systems within the UAV payload are described.

\subsection{The PERIGEO project}

The PERIGEO ${ }^{1}$ project aims to provide a framework for research and validation of space technology and science by means of Earth-analogue environments. Within PERIGEO, tools and methodologies are designed and implemented to continue the efforts performed in laboratory development, exposing the technology to representative and [to a certain extent] realistic environmental and dynamic conditions, similar to those of space missions. This corresponds to increasing the Technological Readiness Level ${ }^{2}$ (TRL) from 3-4, to TRL 5-6 or higher.

Space engineering is probably one of the most challenging and risky technological disciplines due to the extreme conditions encountered in space (temperature, radiation, gravity), which are hardly representative on the Earth. Within a mission, space vehicles face several operation phases until achieving its final destination to perform a particular scientific

${ }^{1}$ Funded by the INNPRONTA 2011-2014 programme (CDTI, Spain)

${ }^{2}$ Quoting European Space Agency (ESA), "TRL $<5$ relate to innovative technologies before/during the mission assessment phase; TRL $>5$ relate to existing technologies and to missions in definition phase.” task e.g. surface planet exploration. From space shuttle launch to orbiting, rendez-vous, or entry, descending and landing, a single set of on-board systems have to face manifold challenges.

Therefore, specific goals in PERIGEO consist in the following: defining new mission and vehicle designs by multidisciplinary optimization of its characteristics; developing a robust and failure-tolerant control system; creating an integrated research and design process to mature the project developments through a logical work sequence (research/laboratory-testing/real-flight-testing); and exploring new navigation methods based on imaging and inertial sensors, including its hybridization. Therefore, PERIGEO paves the way for tests and validation of algorithms and/or methods for space missions.

Four different scenarios are considered within the project: Earth Observation, related to GNC and observation data acquisition and processing from artificial satellites; Interplanetary Flight, focusing on celestial bodies' exploration such as planets or asteroids, aiming at a better understanding of the space characteristics and planetary evolution; Atmospheric Flight, related to the GNC aspects to guide a platform though a planet atmosphere to obtain in-situ measurements from a celestial body including the return to the Earth or other bodies after the exploration; and Entering, Descending and Landing $(E D L)$, in which high precision in navigation and environment characterization are required to safely place the vehicle on the explored body surface.

\subsection{Testing and validation of space systems}

The project defines two testing and validation environments. On one hand, the so-called Dual Synthetic Environment (DSE) facility is devised as a set of hardware and software tools to support the maturation of different methods and technologies related to GNC (Attitude and Orbit Control 
Systems (AOCS), Global Navigation Satellite Systems (GNSS), image processing, etc.) This facility shall act as a demonstrator to test new, particular solutions within real environments, proving its validity and adequacy for final implementation in a production or market phase. With the DSE, validating a new algorithm, or testing a sensor becomes practical and feasible without developing ad-hoc validation frameworks.

On the other hand, the use of Unmanned Aerial Vehicles (UAVs) has been identified as a goal-achieving enabler, particularly for in-flight validation of space-related technologies. The high versatility and accessibility of such platforms permits continuous testing of navigation and control algorithms in close-to-real flight conditions. At this point, a new item might be added to the [exhaustive] list of application niches of UAVs: space technology and science validation.

Many scenarios might then be materialized using these two environments. For example, one scenario that has tailored project developments is that of an atmospheric flight on Titan, the largest moon of Saturn. Atypical profile for that mission is illustrated in Figure 1.

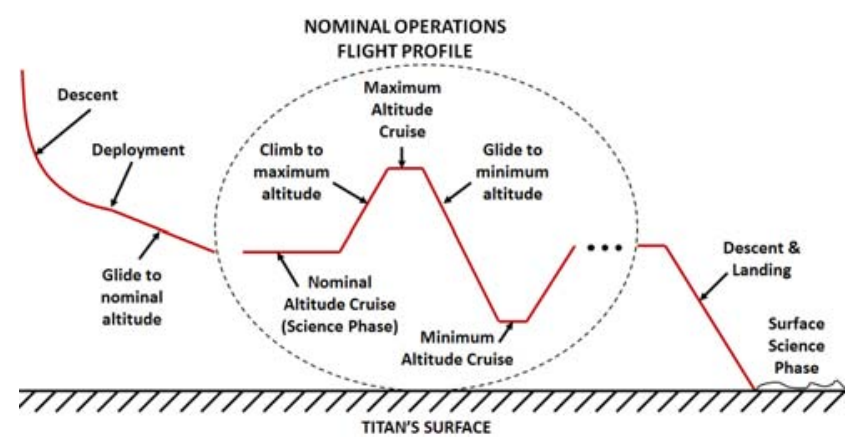

Figure 1: Atmospheric Flight on Titan Mission Profile

In a hypothetical exploration mission, an airplane would be deployed from a space shuttle at around 40 kilometers of altitude to begin exploration i.e. upper-troposphere analysis, while descending to a nominal altitude at which surface observation is feasible (one centimeter of Ground Sampling Distance (GSD) is usually considered). Typically, climb-andglide maneuvers would be performed while transmitting data to Earth ground stations, during continuous observation campaigns that can be as long as months or years, up to final landing of the vehicle to reach the 'in-surface science phase'.

This scenario clearly exemplifies a set of challenges, which are drivers for the developments further explained in this paper, namely:

- Autonomous and real-time navigation. Autonomy in navigation is needed to drive the vehicle along the atmosphere (ground communication to control the aircraft is unaffordable); thus, the navigation solution must be computed and supplied in real-time.

- Unavailability of absolute location beacons. In outer space, the use of GNSS or other type of 'absolute navigation beacons' is simply unfeasible. In this case, navigation is uniquely performed through estimation methods fusing the information obtained independently from external emitters or aiding [Earth re-entry missions, precisely within the 24.000 kilometer height layer, are also considered as atmospheric flight, and exceptionally GNSS measurements might still be used for navigation.]

- Vision-aided INS navigation. In view of the latter, the navigation approach in atmospheric flights shall be based on sensors such as Inertial Measurement Units (IMU), or [passive] imaging systems such as visible-spectrum cameras to deliver time-position-velocity-attitude (tPVA). The use of camera-based measurements aims at replacing the complementary nature of GNSS: compensating for IMU-based solution drifts by calibrating sensor errors.

- Relative and Absolute navigation. Although no radiobeacons might be considered in place, absolute navigation i.e. providing navigation solution referenced to a surfaceattached reference frame, is necessary at some point. For that purpose, the identification of [geo-referenced] surface landmarks e.g. craters, valleys, etc. and the extraction and use of this geo-information for navigation is a common technique in space navigation, and becomes then a goal of the project. Indeed, whenever those landmarks are not available or simply not identified, relative navigation is performed i.e. orientation of consecutive images through exploiting common image features -as a matter of fact, this is 'the only thing to do' when no absolute information is available.

The use of UAVs is of the utmost convenience in relation to the achievement of such goals. The described mission profile is highly replicable using unmanned platforms, and the technology of interest (sensors, systems and algorithms) can be tested on-board, in real payload integration conditions. UAVs bridge the gap between exhaustive laboratory simulation and expensive, ultimate space missions.

\subsection{Review of space navigation: sensors, systems and algorithms}

When considering interplanetary missions, the combination of [on-board] autonomy and [ground-based] automation offers significant advantages in providing the necessary Guidance, Navigation and Control (GNC) and mission science capabilities. The need for autonomy is driven by both mission safety (i.e., quick decisions must be taken for successfully performing the mission), and by cost reduction (i.e. reducing ground-operator work hours). Missions requiring long periods without base contact, and the existence of critical phases i.e. EDL requiring immediate response make communicationdependant GNC simply unfeasible. This fact dictates a high level of on-board autonomy of the spacecraft, covering system, subsystem and instrument level, without forgetting minimum levels of accuracy, precision and robustness.

Nonetheless, and particularly for GNC, Earth-based radioassistance has been traditionally implemented as the main driver of space platforms to reach solar system bodies (Bernhardt et al., 2011). At the end of 1950s, a world-wide network of large antennas and communication facilities called Deep Space Network was established for deep-space vehicle tracking. In addition to radio-assistance, Inertial Measurement Units (IMUs) have been also presents since the rising of space exploration: inertial technology was present the first time mankind left the gravitational influence of Earth i.e. the Apollo 8. Inherited from ballistic rockets, IMUs have been and still are used in top-level missions (Mars Reconnaissance Orbiter, Lunar Reconnaissance Orbiter and Solar TErrestrial RElations Observatory (STEREO) missions included a Miniaturized IMU (MIMU) from Honeywell; and Northrop Grumman's LN200S IMU is present in a wide range of missions, including Mars rovers as the Curiosity).

More and more, the use of other on-board autonomous technology has been fostered. NASA's DeepSpace-1 mission, back in 1998, introduced the Autonomous Optical Navigation (AutoNav) system complementing its on-board IMU. This system, based on triangulation from images of celestial bodies, 
was independent from ground signals as it basically relied on optical cameras as a means of providing measurements for navigation at an affordable cost, size and power consumption. Currently, vision-based systems are essential components of GNC systems in space missions, during all phases of operation. (Massaro, 2013) provides a recent comprehensive review of vision-based spacecraft navigation literature and techniques, with special focus on terrain-relative navigation, demonstrating wide adoption of vision-based solutions in actual systems. Recently, LiDAR technology has also been introduced for space missions, attracted by a superior precision and by its independence from lighting conditions, and particularly as a means to final approach and landing on an asteroid or planet. As a pioneering example, the Hayabusa mission by the Japan Aerospace Exploration Agency used LiDAR technology to successfully land on the Itokawa asteroid in 2005. Current research is available on the use of LiDAR for Terrain Relative Navigation (TRN) and Hazard Detection and Avoidance (HDA) (de Lafontaine et al., 2008), and current technology state-ofthe-art is based on 3D Flash LiDAR technology (Advanced Scientific Concepts, 2013) providing 2D+1D range images.

In parallel to technology, navigation algorithms have had their own evolution in space navigation, all starting from a common origin: the Kalman filter. Following a visit by R.E. Kalman to the NASA office back in 1960 (Grewal and Andrews, 2010), the Apollo mission designers chose a Kalman filter for flying to and back from the Moon, due to its tailoring towards real-time and the ability to cope with non-stationary problems. However, the need to deal with non-linearity and non-Gaussianity conditions, which hold for example in low-fuel missions where nonlinearities are exploited to reduce fuel consumption (Grover and Sato, 2012), have pushed researchers to investigate the use of advance filters e.g. particle filters for space navigation (Ning and Fang, 2008), (Ke et al., 2013).

Despite the criticality of space missions, on-board technology is (sometimes surprisingly) primitive due to limitations on available power on-board, and due to other primary design concerns such as radiation resiliency, faulttolerance in electronics, etc. As an illustrating fact, the Voyager-I platform, the first man-made object launched in 1977 to reach interstellar space, carried three computers able to process 8 kilo-instructions per second -yet, a regular smartphone processes 14 billion of instructions per second. More modern architectures, such as the UT699 LEON3FT SPARC V8 Core, achieve approximately 53 MIPS throughput using a $66 \mathrm{MHz}$ base clock frequency -this performance is comparable to an Intel 486DX, released in 1992. Thus, space developments shall be always constrained to severe hardware limitations, and algorithms and methods on board shall be remarkably 'light'.

\section{NAVIGATION CONCEPT IN PERIGEO}

In view of the context described along the paper, the navigation concept proposed in PERIGEO accounts for a manifold of functionalities:

- The navigation sensor set shall consist on inertial and imaging sensors i.e. optical frame camera. The use of GNSS is restricted to Earth operations e.g. satellite observation, Earth re-entry, etc.

- Absolute and relative navigation modes shall be operational,

- Fault resiliency mechanisms, including hardware and software, shall be in place to deliver robust navigation,
- Algorithms and methods for sensor integration shall be austere in terms of computational burden, mirroring onboard GNC systems, and the scalability of the system shall be characterized to propose upgrades of technology TRL.

Coarsely, relative navigation refers to the propagation of the navigation states (within this paper, time-position-velocityattitude, tPVA) expressed in a convenient reference frame e.g. global, local surface-attached, or local instrumental, between two epochs in which the navigation states have been estimated using external absolute information i.e. absolute navigation, such as previously geo-referenced grounds landmarks or GNSS measurements, if available.

Inertial technology provides inherently relative information -acceleration and rotation rate measurements are differential magnitudes over time. Nonetheless, absolute inertial navigation is achieved simply by numerical integration of the well-known mechanization equations, when expressed in a global reference frame (Rosales and Colomina, 2008). It is also well-known that the inherent relative nature of the measurements is directly translated into drifts over time of the navigation solution -this is why absolute updates e.g. GNSS suitably complement IMUbased navigation.

Now, when using camera images, absolute visual-based navigation is achieved by relating $2 \mathrm{D}$ measurements i.e. extracted from images, with 3D measurements i.e. ground elements. In contrast, when establishing 2D-2D relations between measurements i.e. image-to-image, relative navigation is performed accounting for a particular issue: the scale of the model cannot be retrieved (Horn, 1990). In other words, one degree of freedom of the translation vector between two images is unobservable.

This chapter presents the two proposed scenarios for testing the PERIGEO developments, the simulated environment and the representative environment. For the first scenario, and in order to tailor algorithm development towards real space environments, a tool named Planet and Asteroid Natural Scene Generation Utility (PANGU) has been used to obtain simulated optical images and digital elevation models from the Moon (Rowell et al., 2012). In addition, the OpenCV image processing library (Bradski, 2000) has been used to benefit from low-level handling and processing mechanisms for images. Preliminary results are presented in the simulated environment. For the second scenario, hardware and software developments are integrated into a UAV for real, in-flight testing in representative environments. The first flight campaign will take place during the second half of 2014.

\subsection{Simulated environment: Moon images}

\section{Vision-based relative navigation using point extraction and matching.}

Relative navigation is approached through extraction and matching of particular features of interest in camera images. Every image acquired by the camera is processed in search of distinctive points i.e. pixels that have a particular response in terms of intensity. Once a set of points is extracted from an image at time $t_{n}$, it is matched to a different set of extracted points in a previous image, acquired at time $t_{n-m}$. A large set of literature is available on this topic, resulting in a myriad of methods to perform such task, as reviewed in (Leutenegger et al., 2010). In view of the computational burden restrictions, the use of binary descriptors was considered, resulting on a BRISKbased implementation. 
Once the image measurements are available, the wellknown coplanarity model (Luhmann et al., 2006) is used to solve five degrees of freedom among the set of position and attitude states. More specifically, given the parameters $t=\left(t_{x}, t_{y}, t_{z}\right) \quad$ (the translation vector) and $\Delta \Omega=(\Delta \omega, \Delta \phi, \Delta \kappa)$ (the relative attitude) between two perspective centres from two different, overlapping images, and given the observations $p_{i}^{(1)}, p_{i}^{(2)}, \forall i=1 \ldots N$ (pairs of matched points), a non-linear least-squares problem is formulated using the 'coplanarity' observation equation. Via an iterative approach, estimates of the relative orientation i.e. $(t, \Delta \Omega)$ are provided. [Note that, as one degree of freedom corresponding to the translation vector cannot be estimated, one shall work with conveniently normalized vectors.] The initialization of the leastsquares scheme can be solved by accounting for particular motion characteristics of the platform i.e. forward motion and small attitude variations within epochs.

At this point, robustness is an issue to be dealt with. Automatic image measurement and matching techniques are prone to deliver [a large amount of] outliers i.e. image points that are incorrectly matched. Indeed, environments featuring highly repetitive patterns (urban scenarios, dense forests) or low-textured scenes (planetary surfaces) are conflictive, and shall implement robustness mechanisms.

In PERIGEO, the envisioned strategy is the following: after extracting and matching image points, a prediction of the orientation of the second image is performed (first image is already oriented). Then, the parallax for each matched pair of points i.e. the difference between their ground projections is compared to a chosen threshold. By doing so, outliers and inliers are clearly separated. The aforementioned prediction might be achieved by propagating the navigation states through a low-complexity dynamic model e.g. 'naïve' motion ( $\dot{x}=0, \dot{\Omega}=0$ ), or more complex models e.g. inertial mechanization equations using IMU observables.

Figure 1 depicts matching results using BRISK and descriptor matching by vector comparison using Hammingnorm, and based on the previously described approach i.e. parallax-based outlier removal. This particular results show that 144 out of 386 matched points were actually 'correct' (green lines in the figure, outliers not drawn for clarity), where correctness was defined by a threshold of 0.5 meters in parallax.

\section{Crater-based absolute navigation.}

An important image processing task within the project is crater detection in camera images to approach absolute naviga tion, but also the extraction of craters in LiDAR images aiming at camera/LiDAR co-registration. Craters are common features in outer space bodies, and have been considered as navigation landmarks in literature (van Pham et al., 2010). An algorithm based in contour extraction has been developed to identify crater borders or rims, implemented in two modes: processing camera and LiDAR images. [Note that we refer as 'LiDAR images' to the LiDAR-based digital elevation models, considering 'height' as 'pixel intensity'].

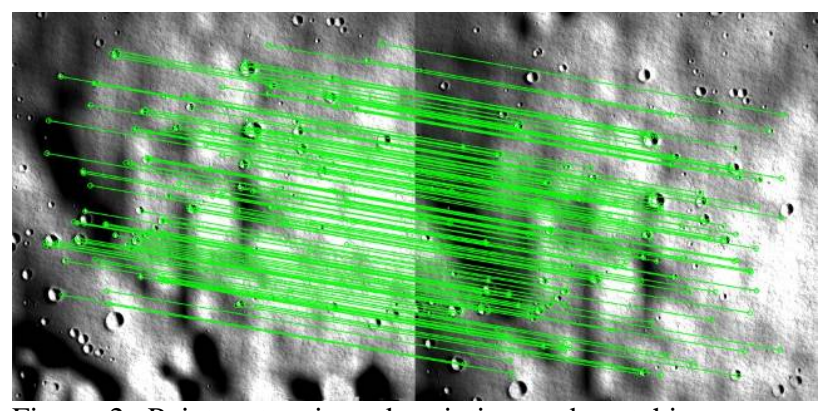

Figure 2. Point extraction, description and matching: correct matched pairs of points in consecutive overlapping images.

For camera images, the algorithm combines the illuminated and non-illuminated parts of the image to identify craters. The current version of the algorithm presents small miss-association issues (Figure 2, leftmost) and few positive detections in low Sun elevation (Figure 2, left-middle) occur -yet, crater detection is feasible regardless of illumination conditions. For the LiDAR images, Sobel gradient operators are applied in the horizontal and vertical directions to extract the variations of the terrain (Figure 2, right-middle). The key fact is that a crater responds to the Sobel operator in all directions, enabling thus its detection (Figure 2, rightmost).

In order to perform absolute navigation, 2D information i.e. extracted craters shall be associated to 3D information i.e. coordinates of the craters in a global frame. For that purpose, a procedure has been developed to describe and match craters. Firstly, to build a descriptor per each group of three craters, encapsulating its local morphology i.e. normalized distances from crater centroids and normalized areas. Secondly, the descriptors are compared and, thanks to the ordination of each group of three craters, a figure of merit for each pair of matched craters is provided.

Note that this procedure is independent of the imaging source from which craters have been extracted. Thus, in the case of absolute navigation, the extracted craters are matched to craters pre-extracted in geo-referenced imagery. By doing so, $3 \mathrm{D}$ coordinates is associated to camera image crater centroids.

At this point, the exterior orientation problem, alternatively known as Perspective n-Point ( $\mathrm{PnP})$ problem, is solved using the approach described in (Garro et al., 2012). The same approach for crater matching is applied to camera and LiDAR crater to perform co-registration.
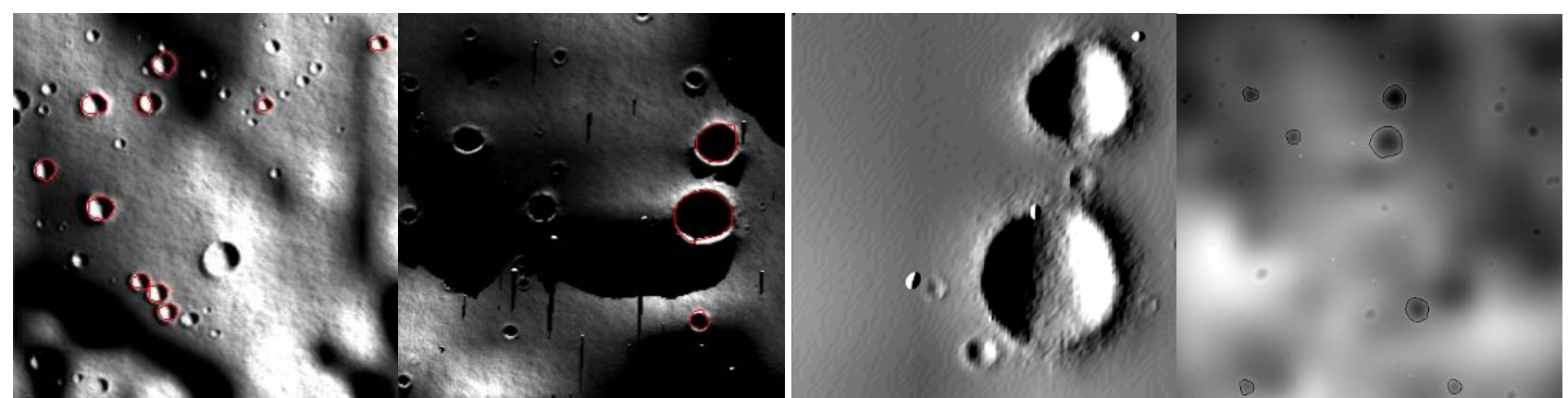

Figure 3. Crater extraction in camera images with Sun elevation around $45^{\circ}$ (leftmost) and Sun elevation around $1^{\circ}$ (left-center); LiDAR image after Sobel operator in the horizontal direction (right-center), and crater identification in LiDAR image (rightmost) 

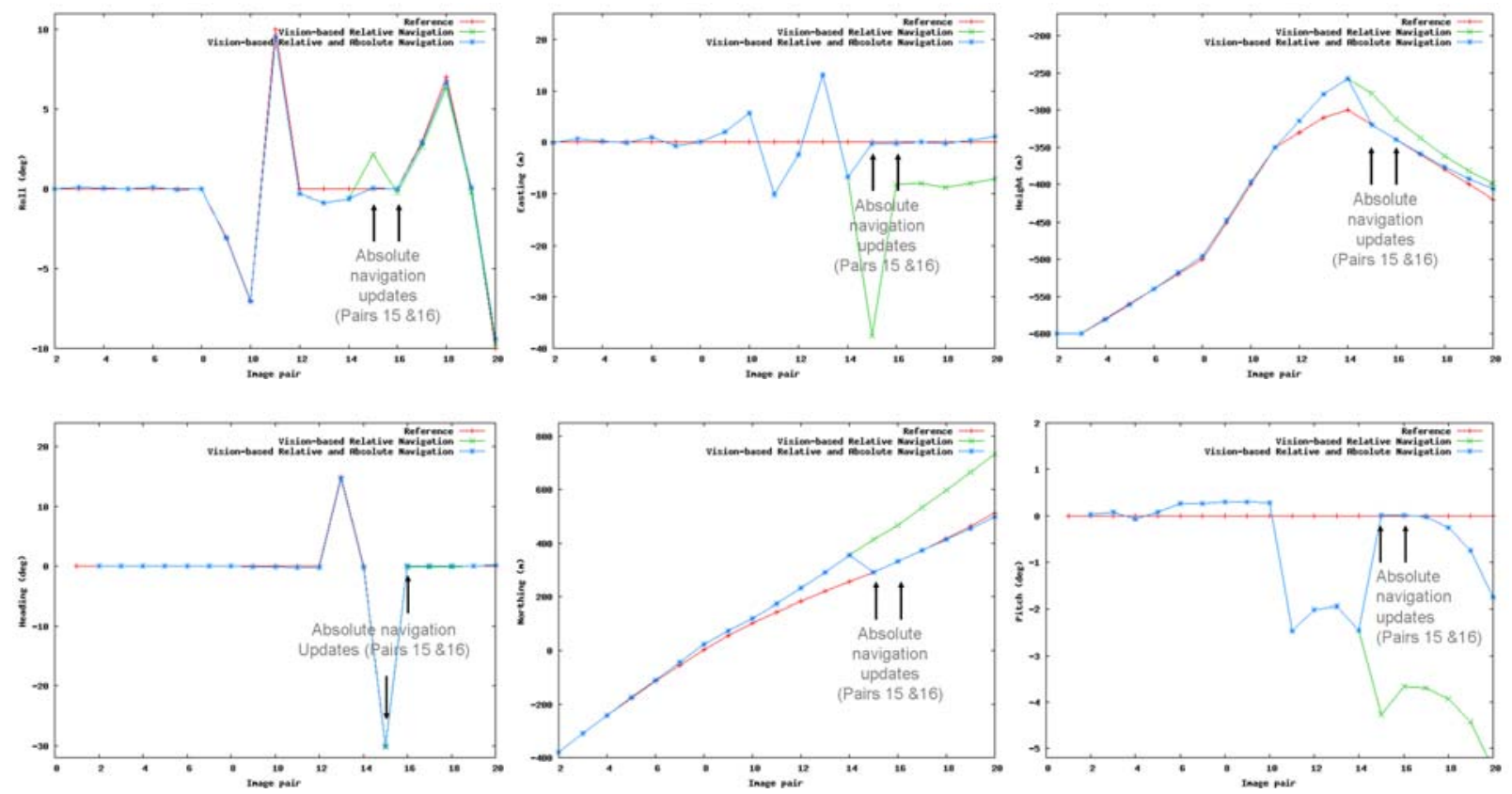

Figure 4. Reference trajectory (red), relative navigation (green) and relative/absolute navigation (blue) results, for northing, easting, height (in meters), and heading, pitch and roll (in degrees), left to right, top to bottom.

\section{Preliminary results for relative and absolute navigation.}

For testing purposes, a trajectory was simulated consisting in forward-motion towards North, 'descend-ascend' manoeuvres, similar to the Titan's flight, with eventual roll and heading turns up to ten and thirty degrees, respectively. A set of 19 simulated image pairs were processed to perform relative and absolute navigation. That is, on one side, point extraction and matching was performed, and known image orientations were used to remove outliers with a parallax threshold of 0.5 meters; and on the other side, craters were extracted from images and, in image pairs 15 and 16, the extracted craters were provided of 3D coordinates in a surface-attached reference frame. Results in position and attitude are provided in Figure 3, comparing a relative navigation solution (green) and a relative/absolute navigation solution (blue), in the terms previously described.

In position estimation for relative navigation, the accumulated along-track and height errors are of $25 \%$ and $15 \%$, respectively. However, the two absolute updates in the relative/absolute trajectory compensates for drifts. Note that the across-track component is correlated with the roll and heading angles, leading to large errors in presence of angle variations for relative navigation. Yet, again due to the absolute updates, this effect is also mitigated for the relative/absolute navigation

On the other hand, attitude is well estimated in both approaches, except for the pitch angle, which is strongly correlated to heading and roll variations. Again, absolute updates are able to decorrelate the parameters and mitigate this effect.

The reader shall keep in mind that these preliminary results are obtained with a camera stand-alone solution, including outlier removal via ideal orientation propagation and fixed parallax threshold. Further research will focus on the integration of inertial measurements for orientation propagation and adaptive thresholding techniques.

\section{Multi-sensor integration for navigation in space processing architectures.}

Another development within PERIGEO is the migration and evaluation of robust estimation algorithms for navigation into a space-like processing architecture. The so-called Fault-Tolerant Processing Architecture (FTPA) is a single-core 32-bit processor LEON3, widely used in space missions, running a RTEMS real-time operating system. Within this processor, the goal of the project is to migrate a multi-sensor, multi-scenario navigation filter, developed by CTTC (Fernández et al., 2010), capable of processing IMU/GNSS or IMU/GNSS/camera sensor configuration, including robustness capabilities. The FTPA is fed through serial ports by other systems providing the navigation measurements.

However, the FTPA features low computation capabilities (a processor power of $50 \mathrm{MHz}$ and $512 \mathrm{~Kb}$ of RAM), supposing a real challenge for algorithm migration. (de Florio et al., 2009) presents a comparative analysis between space processing architectures in an orbit propagation scenario for a satellite in Low Earth Orbit (LEO), including LEON3. The results show that a single update from a Kalman filter implementation, including propagation of position-velocity states and its covariances, takes roughly 1.4 seconds (the interested reader might check further details on the force models or integration times applied in this exercise).

Currently, the migration and adaptation of the navigation filter is being performed through an intermediate processor simulation environment, leading to FTPA-ready software. After the migration, the FTPA will be fed with space-simulated measurements (IMU and camera) within the DSE to reproduce realistic space conditions.

\subsection{Representative environment: integration in the UAV}

Project testing is a key component in PERIGEO, as the project seeks to increase the TRL of the developed technologies using UAVs as a means for this purpose. Table 1 shows the subsystems and sensors to be integrated in the UAV payload.

\begin{tabular}{|c|c|}
\hline $\begin{array}{l}\text { Subsystem or } \\
\text { sensors }\end{array}$ & \multicolumn{1}{c}{ Functionalities } \\
\hline GRIP & $\begin{array}{l}\text { In-house multi-frequency GNSS receiver, } \\
\text { featuring Galileo's AltBOC signal reception }\end{array}$ \\
\hline
\end{tabular}


The International Archives of the Photogrammetry, Remote Sensing and Spatial Information Sciences, Volume XL-3/W1, 2014 EuroCOW 2014, the European Calibration and Orientation Workshop, 12-14 February 2014, Castelldefels, Spain

\begin{tabular}{|c|l}
\hline VISIONA & $\begin{array}{l}\text { Camera- and LiDAR-based navigation } \\
\text { system, and input provider for HDA tasks }\end{array}$ \\
\hline CAPTURA & $\begin{array}{l}\text { Miniaturized INS/GNSS acquisition system } \\
\text { and time-reference server }\end{array}$ \\
\hline $\begin{array}{c}\text { Fault-tolerant } \\
\text { Processing } \\
\text { Architecture }\end{array}$ & $\begin{array}{l}\text { LEON3-based processing architecture } \\
\text { including INS/GNSS/image processing } \\
\text { software }\end{array}$ \\
\hline $\begin{array}{c}\text { Prosilica } \\
\text { GC2450C }\end{array}$ & Camera for online image processing tasks \\
\hline $\begin{array}{c}\text { Hokuyo UTM- } \\
\text { 30LX }\end{array}$ & $\begin{array}{l}\text { LiDAR for online digital elevation model } \\
\text { generation }\end{array}$ \\
\hline Sony NEX-5N & Camera for high-resolution observation \\
\hline $\begin{array}{c}\text { EPSON M- } \\
\text { G350-PD11 }\end{array}$ & Low-cost miniaturized IMU \\
\hline Javad TR-G3T & $\begin{array}{l}\text { Geodetic-grade, multi-frequency GNSS } \\
\text { receiver }\end{array}$ \\
\hline
\end{tabular}

Table 1. Subsystems and sensors integrated in the UAV

Genuine space sensors and systems are not available within the project -thus, the scalability and restrictions of the previously discussed requirements have been analyzed and the appropriate technologies have been selected to be tested inflight. As an example, the short LiDAR sensor scanning range is regarded as a 'scale problem', in which cost and performance are clearly correlated. In this case, modulating the mission flying altitude enables the use of LiDAR within the project. Figure 5 shows particular developments of the CTTC within PERIGEO, and sensors to be integrated.
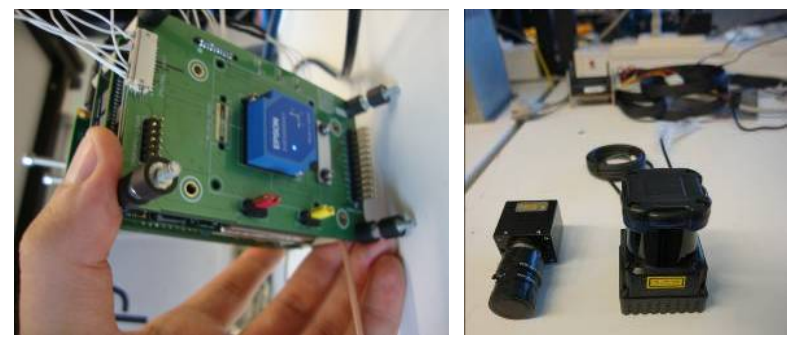

Figure 5. EPSON IMU M-G350-PD11 mounted on CAPTURA (left); Hokuyo LiDAR and Prosilica camera, to be interfaced in VISIONA (right)

The UAV to be used for project testing is the SIVA (Figure 6), a fixed-wing platform developed by Instituto Nacional de Tecnologia Aeroespacial (INTA), featuring $300 \mathrm{~kg}$ of MTOW, 6 hours of endurance, a maximum speed of $170 \mathrm{~km} / \mathrm{h}$, and able to carry $40 \mathrm{~kg}$ of payload.

Project testing shall be conducted around a controlled and segregated area around a take-off and landing site in which SIVA is authorized to manoeuvre e.g. regional airports. Depending on the final testing site, the analogies with outer space scenarios is not obvious, and therefore, preparation of the test site is mandatory to include space-like elements i.e. craters. Further testing shall consider areas with a high-degree of likeliness with the Moon or other bodies of interest.

\section{SUMMARY AND WAY FORWARD}

This paper presents the current status of developments in relation to navigation tasks within the PERIGEO project. The paper has presented the context of space navigation, highlighting the limitations and requirements of sensors and systems for such purpose. After that, the two testing environments of the project have been presented. Firstly, within the simulation environment, Moon images have been simulated and preliminary results have been presented for relative and ab-
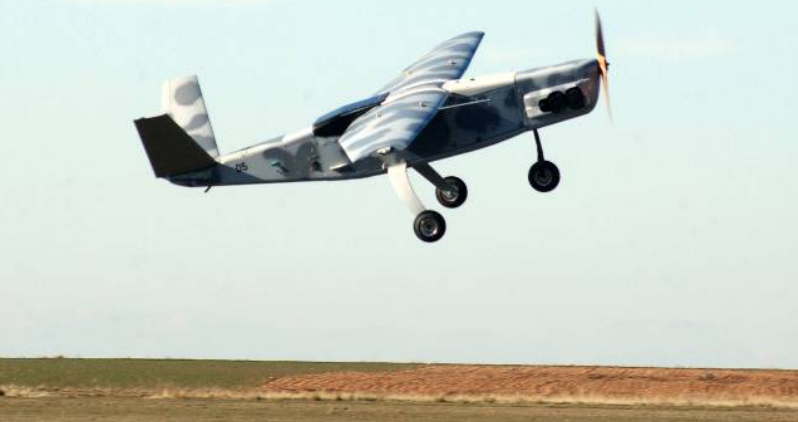

Figure 6. The SIVA unmanned aerial vehicle (INTA)

solute navigation, based on point and crater extraction from images, respectively. Secondly, the representative environment consists on hardware/software integration into a UAV payload, including cameras, LiDAR and IMU, to perform in-flight testing of the development during the current year.

Further research in PERIGEO will focus on the robustness for vision-based navigation, by analyzing the potential of parallax-based outlier rejection with relative orientation propagation using various dynamic models. The final phase of the project will also cover extensive result generation for the developed algorithms.

\section{REFERENCES}

Advanced Scientific Concepts, 2013. DragonEye 3D Flash LIDAR Space Camera, accessed through the web on February, 2014.

Bernhardt, M.G., Becker,W., Prinz, T., Breithuth,F.M., Walter,U., 2011. Autonomous Spacecraft Navigation Based on Pulsar Timing Information, Proceedings of the 2nd International Conference on Space Technology, 15-17 Sept. 2011, Athens, Greece.

Bradski, G., 2000. The OpenCV Library. Dr. Dobb’s Journal of Software Tools, 2000.

De Florio, S., Gill, E.,. D'Amino, S., Grillenberger, A., 2009. Performance Comparison of Microprocessors for Space-Based Navigation Applications, Proceedings of 7th IAA Symposium on Small Satellites for Earth Observation, May 2009.

De Lafontaine, J., Neveu, D., Hamel, J-F, 2008. Autonomous planetary Lansing using a LiDAR sensor: the Landing Dynamic Test Facility, Proceedings of the $7^{\text {th }}$ ESA conference on Guidance, Navigation and Control Systems, 2-5 June 2008, County Kerry, Ireland.

Fernández, A., Diez, J., Castro, D.D., Dovis, F., Silva, P., Friess, P., Wis, M., Colomina, I., Lindenberger, J., Fernández, I., 2010. ATENEA: Advanced Techniques for Deeply Integrated GNSS/INS/LiDAR Navigation. Proceedings of the 5th ESA Workshop on Satellite Navigation Technologies, Navitec 2010, Noordwijk, The Netherlands, December 2010.

Garro, V., Crosilla, F., Fusiello, A., 2012. Solving the PnP problem with anisotropic orthogonal procrustes analysis. Proceedings of the Second Joint 3DIM/3DPVT Conference: 3D 
The International Archives of the Photogrammetry, Remote Sensing and Spatial Information Sciences, Volume XL-3/W1, 2014

EuroCOW 2014, the European Calibration and Orientation Workshop, 12-14 February 2014, Castelldefels, Spain

Imaging, Modeling, Processing, Visualization and Transmission 3DIMPVT 2012, pages 262-269, 2012.

Grewal, M. and Andrews, A., 2010. Applications of Kalman Filtering in Aerospace 1960 to the Present IEEE Control Systems Magazine, Vol. 30, No. 3. (June 2010), pp. 69-78, doi:10.1109/mcs.2010.936465

Grover, P., Sato, Y., 2012. Efficient Estimation and Uncertainty Quantification in Space Mission Dynamics, AIAA/AAS Astrodynamics Specialist Conference, DOI: 10.2514/6.20125062, August 2012

Horn, B.K.P., 1990. Recovering baseline and orientation from essential matrix. Journal of Optical Society of America, 1990.

Ke, L., Qian, Z., Bin, G., Wei, L., 2013. A Novel Particle Filter Algorithm and Its Application in Relative Navigation in Spacecraft Rendezvous and Docking. Manned Space Flight, Issue 5. China Manned Space Agency, 2013.

Leutenegger, S., Chli, M., Siegwart, R., 2011. BRISK: Binary Robust Invariant Scalable Keypoints, Proceedings of the IEEE International Conference on Computer Vision (ICCV) 2011.

Luhmann, T., Robson, S., Kyle, S., Harley, I., 2006. Close Range Photogrammetry: Principles, Methods and Applications. Published by Whittles Publishing. ISBN 1870325508
Massaro, A., 2013. Synthesis and Validation of Vision Based Spacecraft Navigation, PhD Dissertation, Technical University of Denmark DTU Space - National Space Institute, 2013.

Ning, X., and Fang, J., 2008. Spacecraft autonomous navigation using unscented particle filter-based celestial/Doppler information fusion. Measurement Science and Technology, 19 doi:10.1088/0957-0233/19/9/095203.

Rosales, J.J., Colomina, I., 2005. A flexible approach for the numerical solution of the INS mechanization equations, Proceedings of the 6th Geomatic week, Barcelona, 2005

Rowell, N., Parkes, S., Dunstan,M., Dubois-Matra, O., 2012. PANGU: Virtual spacecraft image generation. Proceedings of the 5th International Conference on Astrodynamics Tools and Techniques, ICATT 2012, ESA ESTEC, Noordwijk, The Netherlands, 2012

Van Pham, B., Lacroix, S., Devy, M., Drieux, M., Voirin, T., 2010. Landmark Constellation Matching for Planetary Lander Absolute Localization. Proceedings of the $5^{\text {th }}$ International Conference on Computer Vision Theory and Applications, Angers, France, May 17-21, 2010. 